\title{
COVID-19 e a Odontologia na prática atual
}

\author{
COVID-19 and Dentistry in current practice \\ COVID-19 y Odontología en la práctica actual
}

Kátia Ferreira dos Santos ${ }^{1 *}$, Marcelo Barbosa².

\begin{abstract}
RESUMO
Objetivo: Pesquisar medidas preventivas recomendadas, com a finalidade de diminuir o risco de infecção e contaminação cruzada, em clínicas e consultórios odontológicos públicos e/ou privados, no período da pandemia. Métodos: Revisão integrativa da literatura, com busca recente na base de dados PubMed/MEDLINE (Medical Literature Analysis and Retrieve System Online), que é uma fonte de informação multidisciplinar na literatura biomédica, com indexação de artigos de revistas em nível mundial. Foram utilizados os seguintes termos de busca para esta pesquisa, em inglês: "COVID-19 and Dentistry" (Coronavirus Disease-2019 e odontologia) e os critérios de inclusão foram: artigos na língua inglesa, com versão completa on-line, gratuita e aqueles que ainda se encontram em vias de publicação. Resultados: Foram encontrados 35 artigos relacionados com o tema, todos com título e resumo em inglês. Desses, 20 não correspondiam diretamente ao tema, somente 13 artigos atendiam aos critérios de inclusão que foram selecionados e analisados. Considerações finais: Práticas biosseguras, aferição de temperatura corpórea e adequação aos testes para COVID-19 podem ser práticas inseridas, no contexto odontológico, para ajudar no combate a pandemia.
\end{abstract}

Palavras-chave: Infecções por coronavírus, Odontologia em saúde pública, Controle de infecções, Saliva, Contenção de riscos biológicos.

\begin{abstract}
Objective: To research recommended preventive measures, with the purpose of reducing the risk of infection and crosscontamination, in public and / or private dental clinics and offices, during the pandemic period. Methods: Integrative literature review, with a recent search in the PubMed / MEDLINE (Medical Literature Analysis and Retrieve System Online) database, which is a source of multidisciplinary information in biomedical literature, with indexing of journal articles worldwide. The following search terms were used for this search, in English: "COVID-19 and Dentistry" (Coronavirus disease-2019 and Odontologia), and the inclusion criteria were: articles in English, with free online full version and those that are still being published. Results: 35 articles were found related to the theme, all with title and abstract in English. Of these, 20 did not correspond directly to the theme, only 13 articles met the inclusion criteria that were selected and analyzed. Final considerations: Biosafety practices, measurement of body temperature and adaptation to tests for COVID-19 can be inserted practices, in the dental context, to help in fighting the pandemic.
\end{abstract}

Keywords: Coronavirus infections, Public health dentistry, Infection control, Saliva, Containment of biohazards.

\section{RESUMEN}

Objetivo: Investigar las medidas preventivas recomendadas, con el propósito de reducir el riesgo de infección y contaminación cruzada, en clínicas y consultorios dentales públicos y / os privados, durante el período pandémico. Métodos: Revisión integradora de la literatura, con búsqueda reciente en la base de datos PubMed / MEDLINE (Medical Literature Analysis and Retrieve System Online), que es una fuente de información multidisciplinaria en la literatura biomédica, con indexación de artículos de revistas de todo el mundo. Para esta búsqueda se utilizaron los siguientes términos de búsqueda, en inglés: "COVID-19 and Dentistry" (Coronavirus Disease-2019 y Odontología), y los criterios de inclusión fueron: artículos en inglés, con versión completa online gratuita y los que aún se están publicando. Resultados: Se encontraron 35 artículos relacionados con el tema, todos con título y resumen en inglés. De estos, 20 no correspondían directamente a la temática, solo 13 artículos cumplieron con los criterios de inclusión que fueron seleccionados y analizados. Consideraciones finales: Las prácticas de bioseguridad, medición de la temperatura corporal y adaptación a pruebas para COVID-19 pueden insertarse prácticas, en el contexto odontológico, para ayudar en la lucha contra la pandemia.

Palabras-clave: Infecciones por coronavirus, Odontología en salud publica, Control de infecciones, Saliva, Contención de riesgos biológicos.

${ }^{1}$ Programa de Pós-Graduação em Ciências da Coordenadoria de Controle de Doenças da Secretaria de Estado da Saúde de São Paulo, São Paulo - SP. *E-mail: katiasantosdentista@gmail.com

2 Instituto de Infectologia Emílio Ribas, São Paulo - SP. 


\section{INTRODUÇÃO}

No final do ano de 2019, na província de Hubei, na cidade de Wuhan, localizada na China Continental, surgiu o novo coronavírus, até então chamado de $2019-\mathrm{nCoV}$, pertencente à linhagem $\mathrm{B}$ do gênero Betacoronavírus, relacionado ao coronavírus do morcego e responsável pela síndrome respiratória aguda grave (SARS), porém as diferenças estão na forma eficiente de transmissão entre os humanos e como o vírus se espalhou pelo planeta gerando uma pandemia.

A detecção rápida do 2019-nCoV é importante para o controle do surto na comunidade e também para os profissionais de saúde, pois algumas características deste vírus ainda não são bem conhecidas (TO KKW, et al., 2020). Os vírus de maiores problemas são o SARS-CoV que causa a síndrome respiratória aguda grave e o coronavírus da síndrome respiratória do Oriente Médio, também conhecido por MERS-CoV, ambos de origem zoonótica e muitas vezes associadas a doenças fatais.

O 2019-nCoV-2 é o sétimo membro da família dos coronavírus que infectam seres humanos (ZHU N, et al.,2020). A partir de 11 de fevereiro de 2020, a World Health Organization (WHO) passou a chamar a infecção pelo 2019-nCov, como Coronavirus Disease-2019 (COVID-19) e, a partir de 11 de março de 2020 a Pandemia foi declarada.

Os sintomas da infecção por COVID-19 incluem febre, tosse seca, falta de ar evoluindo para doença respiratória aguda, com casos graves levando a pneumonia, insuficiência renal e até morte, rapidamente. Recentemente, testes salivares do COVID-19 em humanos revelaram a presença do vírus (SABINO-SILVA $\mathrm{R}$, et al., 2020).

A Johns Hopkins University (2020) alertou em 10/04/2020 que o número de pessoas atingidas pela COVID19 ultrapassou um milhão, com um total de 1.733 .792 e um total de mortos que chegam a 106.469 mil pessoas, sendo os países mais atingidos: Itália, com 19.469, Espanha, com 16.353, França, com 13.197, Reino Unidos, com 5.820 e a cidade de Nova York, nos Estados Unidos, com 5.820.

O Brasil ocupou o $11^{\circ}$ lugar, com um total de 1.075 mortos e 20.022 casos confirmados com a doença. Em pouco mais de quatro meses, aproximadamente 130 dias, o Brasil passou a ocupar o $2^{\circ}$ lugar com 3.908.272 casos confirmados e a $2^{\underline{a}}$ posição de óbitos, totalizando 121.381 mortos (JOHNS HOPKINS UNIVERSITY, 2020). A transmissão do vírus acontece por inalação, ingestão e/ou contato direto das mucosas com gotículas de saliva. Vale ressaltar que o vírus pode sobreviver nas mãos, objetos ou superfícies expostas à saliva infectada por vários dias (SPAGNUOLO G, et al., 2020).

Há três formas diferentes do novo coronavírus, na saliva, causar a infecção: a primeira é pela presença do vírus no trato respiratório inferior e superior que pode entrar na cavidade oral, com as gotículas de líquido trocadas por esses órgãos. A segunda, quando o vírus presente no sangue, pode acessar a boca, via fluido do sulco gengival (crevicular), um exsudato específico da cavidade oral, e a terceira é por infecção das glândulas salivares maiores e menores, com liberação subsequente de partículas, na saliva, por dutos salivares (SABINO-SILVA R, et al., 2020).

Um dos meios de detecção da COVID-19 são os testes salivares, realizados por meio de cultura viral, que podem reduzir a chance de contaminação entre as pessoas e, principalmente, entre os profissionais da saúde, por serem de fácil acesso, não invasivos, com resultado rápido e economicamente viável (TO KKW, et al., 2020). Spagnuolo G, et al. (2020) descreveram os cirurgiões-dentistas, como os trabalhadores mais expostos ao risco de serem afetados pela doença, mais do que enfermeiros e clínicos gerais. Um artigo escrito por pesquisadores da Escola Wuhan University e Hospital de Estomatologia foi publicado com várias recomendações para dentistas e estudantes de odontologia com a finalidade de gerenciar pacientes portadores do COVID-19 (MENG L, et al., 2020).

O primeiro caso de um dentista com teste positivo, para COVID-19, foi relatado no final do mês de janeiro de 2020, pelo Departamento de Odontologia Preventiva do Hospital Dentário da Universidade de Wuhan, posteriormente, foram identificados oito profissionais da saúde bucal, contaminados pela disseminação da doença (MALLINENI SK, et al., 2020). Medidas de biossegurança devem fazer parte deste cenário pandêmico como uma forma de "prevenir, controlar, mitigar ou eliminar riscos inerentes às atividades que possam 
interferir ou comprometer a qualidade de vida, a saúde humana e o meio ambiente" (BRASIL e ORGANIZAÇÃO PANAMERICANA DA SAÚDE, 2010). Coulthard P (2020) enfatiza que o uso de máscaras cirúrgicas está indicado como medida protetiva, pois oferece filtragem de até $80 \%$ em procedimentos eletivos das partículas aerossolizadas presentes no ambiente odontológico.

Os aerossóis são partículas líquidas e sólidas suspensas no ar, por um período de tempo, são menores do que $50 \mu \mathrm{m}$ (micrômetros) de diâmetro e podem se formar pelo uso de instrumentos rotatórios odontológicos. É recomendado o uso dos equipamentos de proteção individual (EPI) e evitar procedimentos que produzam gotículas ou aerossóis, bem como, o uso de ejetores de saliva, com bomba a vácuo que ajudem na redução destes contaminantes (SPAGNUOLO G, et al., 2020).

O splatter, conhecido por respingos, é uma mistura de ar, água e/ou substâncias sólidas com tamanho de $50 \mu \mathrm{m}$ a vários milímetros de diâmetros e apresentam riscos de contaminação aos trabalhadores da odontologia, pois podem conter bactérias, vírus, fungos e sangue quando associado aos instrumentos rotatórios.

Tendo em vista que o coronavírus mede cerca de $120 \mathrm{~nm}$ (nanômetros) ou $0.12 \mu \mathrm{m}$ e a partícula de aerossol mede de 3-100 nm, está indicado o uso de máscaras descartáveis do tipo FFP3 (peça facial filtrante de nível 3, de acordo com as normas europeias), pois oferece uma taxa de filtragem em torno de $99 \%$ de partículas que medem $0.6 \mu \mathrm{m}$ e é a mais indicada como EPI, evita a contaminação cruzada e permite a continuidade das urgências/emergências odontológicas (COULTHARD P, 2020). Os enxaguatórios e antissépticos bucais podem ser indicados para ajudar a reduzir uma porção infecciosa, mas não eliminam o vírus da saliva humana (SPAGNUOLO G, et al., 2020). No estudo de Caruso AA, et al. (2020) a água oxigenada $\left(\mathrm{H}_{2} \mathrm{O}_{2}\right)$ volume 10 a $3 \%$ é indicada para desinfecção da cavidade oral e em casos de gengivite.

A indicação em forma de gargarejo, pura, 3 vezes ao dia e em lavagens nasais com uso de nebulizador, 2 vezes ao dia, sem que haja danos nas mucosas orais e seus microvilos funciona como coadjuvante, na oxidação de vírus e também, está indicado em casos leves e moderados de COVID-19. A iodopovidona a 0,5\%-0,6\% é indicada como colírio, em infectados. Esses procedimentos reduzem o número de internações e complicações respiratórias causadas pela COVID-19, principalmente no início da infecção.

Desde março de 2020, a American Dental Association (ADA) atualizou a comunicação com pacientes e profissionais, oferecendo um treinamento via online, para orientar o atendimento odontológico e facilitar o trabalho de forma segura para ambos (WHO, 2020). As medidas da WHO no combate à pandemia são: urgências odontológicas, diminuição do contato interpessoal, tempo reduzido na sala de espera, abordagem com perguntas sobre o estado de saúde geral do paciente nos últimos 7 dias, sobre o risco de ter contato com outras pessoas infectadas e verificação da temperatura maior que $37,5^{\circ} \mathrm{C}$, antes de se iniciar $\mathrm{O}$ tratamento (SPAGNUOLO G, et al., 2020).

O Brasil seguiu as recomendações da WHO quanto à indicação de urgências e emergências odontológicas, com a finalidade de não deixar a população sem acesso aos serviços (BRASIL, 2020). Para Caruso AA, et al. (2020) o uso de peróxido de hidrogênio $\left(\mathrm{H}_{2} \mathrm{O}_{2}\right)$ a $0,5 \%$ é indicado em superfícies de ambiente hospitalar, salas cirúrgicas e também em ambientes odontológicos, como métodos de higienização e desinfecção.

As superfícies inanimadas podem permanecer infectadas pelos coronavírus de 2 horas até nove dias. 0 uso de hipoclorito de sódio de superfície a $0,1 \%$ é indicado como agente de desinfecção e, também, o uso de etanol, com tempo de exposição de 1 minuto, na concentração de 62 a $71 \%$ mostrou, neste estudo, uma redução significativa da infectividade do coronavírus nas superfícies (KAMPF G, et al., 2020). A WHO (2014) recomenda o uso de etanol a 70\% para desinfecção de superfícies.

Cabe ressaltar que, por meio de conhecimentos científicos associados à biossegurança, controle de limpeza de superfície de trabalho e de material biológico, a prática odontológica pode ser exercida com menos riscos a saúde do profissional e dos pacientes. O objetivo deste artigo é pesquisar, por meio de revisão de literatura atual e informar, os cirurgiões dentistas, para a atuação clínica de urgência e emergência, vias de transmissão da COVID-19, possíveis testes para detecção da doença e medidas preventivas utilizadas, para diminuir o risco de infecção nos profissionais atuantes e no ambiente odontológico. 


\section{MÉTODOS}

Foi realizada uma revisão integrativa com uma busca na base de dados PubMed/MEDLINE: fonte de informação multidisciplinar na literatura biomédica com indexação de artigos de revistas em nível mundial, diretamente com os termos de busca, em inglês: "COVID-19 and Dentistry" (Coronavirus Disease-2019 e odontologia), no período de 20/03/2020 a 14/04/2020. Para o critério de inclusão foram utilizados os artigos na língua inglesa, que apresentavam a versão completa, on-line, gratuita e também aqueles que ainda se encontram em vias de publicação (Epub ahead of print). Os critérios de exclusão se restringiram àqueles que, após a leitura do título e resumo, não apresentavam correlação direta com os termos de busca e/ou foram escritos em outro idioma (Figura 1).

Figura 1 - Fluxograma representando os critérios de busca na literatura e inclusão de artigos.

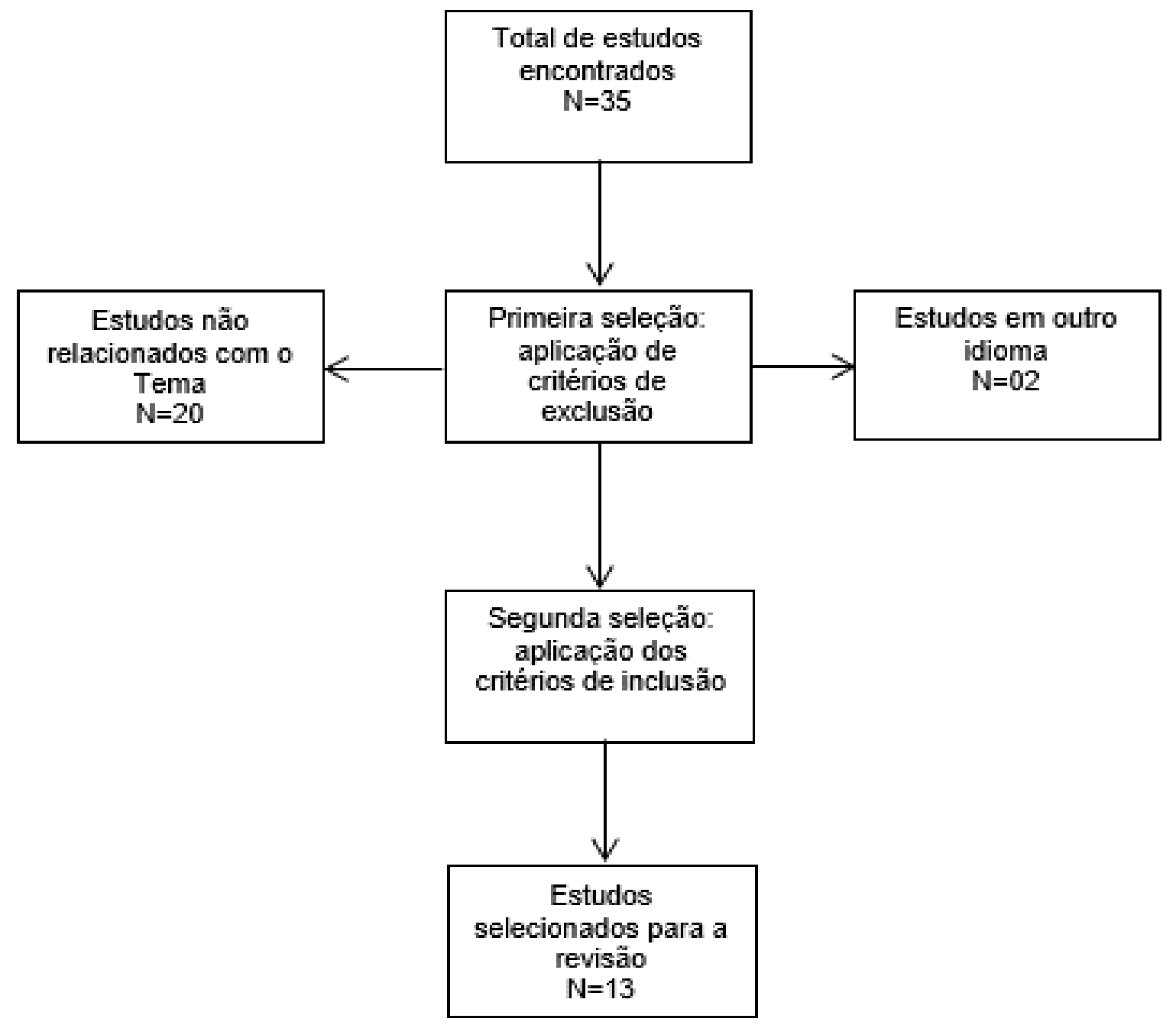

Fonte: Santos KF e Barbosa M, 2020.

\section{RESULTADOS}

Foram encontrados, na busca com palavras-chave, um total de 35 artigos, todos com título e resumo em inglês, de acordo com as normas do PubMed, com acesso gratuito, online, na versão completa e em vias de publicação. Desses, 20 não correspondiam diretamente ao tema, sobrando 15 artigos. Dos 15 remanescentes, 2 artigos, embora apresentassem os critérios de inclusão acesso gratuito, online, versão completa e o tema, o idioma não correspondia à língua inglesa, sendo assim retirados do estudo. Restaram 13 artigos que atendiam a todos os critérios de inclusão que foram selecionados e analisados. Em todos os artigos selecionados foram identificadas medidas preventivas adotadas seguindo sugestões dos órgãos oficiais e recomendações referendadas (Quadro 1). 
Quadro 1 - Artigos selecionados com medidas preventivas e recomendações.

\begin{tabular}{|c|c|c|c|}
\hline Autor & $\begin{array}{l}\text { País do } \\
\text { Estudo* }\end{array}$ & $\begin{array}{c}\text { Medidas preventivas adotadas } \\
\text { nos estudos }\end{array}$ & Recomendações sugeridas \\
\hline $\begin{array}{l}\text { Prati C, et al., } \\
2020\end{array}$ & A & $\begin{array}{l}\text { Triar, separar e evitar tratamentos } \\
\text { eletivos odontológicos, para evitar } \\
\text { contaminações. }\end{array}$ & $\begin{array}{c}\text { Indicação de lavagem das } \\
\text { mãos, indicação de testagem, } \\
\text { com monitoramento da COVID- } \\
19 .\end{array}$ \\
\hline $\begin{array}{l}\text { Coulthard P, } \\
2020\end{array}$ & B & $\begin{array}{c}\text { Uso de máscaras cirúrgicas para } \\
\text { profissionais, pacientes com tosse e } \\
\text { N95, se necessário. }\end{array}$ & $\begin{array}{c}\text { Seguir as diretrizes da OMS e } \\
\text { usar os EPI. }\end{array}$ \\
\hline $\begin{array}{l}\text { Casamassimo } \\
\text { PS, et al., } 2020\end{array}$ & C & $\begin{array}{c}\text { Tratamento de emergência e } \\
\text { urgência de acordo com questões } \\
\text { éticas da odontopediatria, com uso } \\
\text { de EPI. }\end{array}$ & $\begin{array}{l}\text { Rediscutir desafios éticos de } \\
\text { saúde e fiscais como estratégia } \\
\text { de educação em saúde. }\end{array}$ \\
\hline $\begin{array}{l}\text { Caprioglio A, et } \\
\text { al., } 2020\end{array}$ & A & $\begin{array}{l}\text { Seguir as diretrizes da OMS e } \\
\text { autoridades locais com } \\
\text { questionários de rastreamento, } \\
\text { seguido do uso de EPI. }\end{array}$ & $\begin{array}{l}\text { Assistência virtual, por meio do } \\
\text { Whatsapp, como uma forma de } \\
\text { comunicação, para minimizar } \\
\text { urgências ortodônticas. }\end{array}$ \\
\hline $\begin{array}{l}\text { Khader Y, et al., } \\
2020\end{array}$ & $\mathrm{D}$ & $\begin{array}{l}\text { Seguir as normas e diretrizes do } \\
\text { CDC e da ADA baseado nas } \\
\text { recomendações, da situação local. }\end{array}$ & $\begin{array}{c}\text { Medidas de prevenção e } \\
\text { controle de infecções e o } \\
\text { adiamento de tratamentos, não } \\
\text { emergenciais. }\end{array}$ \\
\hline $\begin{array}{l}\text { Mallineni SK, et } \\
\text { al., } 2020\end{array}$ & E & $\begin{array}{c}\text { Acatar as medidas de segurança } \\
\text { local, regional e nacionalmente } \\
\text { relevantes, seguido de proteção às } \\
\text { crianças afetadas. }\end{array}$ & $\begin{array}{l}\text { Uso de precaução universal } \\
\text { quando o tratamento dental } \\
\text { estiver indicado, para crianças. }\end{array}$ \\
\hline $\begin{array}{l}\text { Martelli-Júnior H, } \\
\text { et al., } 2020\end{array}$ & $\mathrm{~F}$ & $\begin{array}{l}\text { Reflexão por meio de trabalhos } \\
\text { científicos, para atualizar e } \\
\text { disseminar conhecimentos. }\end{array}$ & $\begin{array}{c}\text { Aconselhamento, por meio das } \\
\text { histórias das pandemias } \\
\text { anteriores. }\end{array}$ \\
\hline $\begin{array}{l}\text { Farooq I, Ali S, } \\
2020\end{array}$ & G & $\begin{array}{c}\text { Priorizar atendimento odontológico } \\
\text { de emergência e evitar tratamentos } \\
\text { eletivos. }\end{array}$ & $\begin{array}{c}\text { Apoio financeiro aos } \\
\text { consultórios odontológicos. }\end{array}$ \\
\hline $\begin{array}{l}\text { Berlin-Broner Y, } \\
\text { Levin L, } 2020\end{array}$ & $\mathrm{H}$ & $\begin{array}{c}\text { Tratamentos baseados na } \\
\text { hierarquia das necessidades } \\
\text { odontológicas. }\end{array}$ & $\begin{array}{c}\text { Hierarquia das necessidades } \\
\text { dentais para eleger prioridades } \\
\text { de tratamento. }\end{array}$ \\
\hline $\begin{array}{l}\text { Spagnuolo G, et } \\
\text { al., } 2020\end{array}$ & A & $\begin{array}{l}\text { Uso de EPI e boa comunicação } \\
\text { entre profissionais e pacientes. }\end{array}$ & $\begin{array}{c}\text { Atender as urgências } \\
\text { odontológicas com verificação } \\
\text { de temperatura, informações } \\
\text { sobre estado de saúde geral } \\
\text { dos últimos } 7 \text { dias. }\end{array}$ \\
\hline $\begin{array}{l}\text { Yang Y, et al., } \\
2020\end{array}$ & I & $\begin{array}{l}\text { Prevenção e controle de doenças } \\
\text { na área bucomaxilofacial. }\end{array}$ & $\begin{array}{l}\text { Seguir as recomendações do } \\
\text { Ministério da Saúde da } \\
\text { República Chinesa e categorizar } \\
\text { as urgências, em cirurgia } \\
\text { bucomaxilofacial. }\end{array}$ \\
\hline $\begin{array}{l}\text { Peng } X \text {, et al., } \\
2020\end{array}$ & I & $\begin{array}{c}\text { Medidas preventivas com controle } \\
\text { de temperatura, higiene das mãos e } \\
\text { uso EPI. }\end{array}$ & $\begin{array}{c}\text { Seguir as recomendações } \\
\text { baseadas nas diretrizes oficiais } \\
\text { para diagnóstico e tratamento } \\
\text { de novas pneumonias por } \\
\text { coronavírus. }\end{array}$ \\
\hline $\begin{array}{l}\text { Sabino-Silva R, } \\
\text { et al., } 2020\end{array}$ & $J$ & $\begin{array}{l}\text { Buscar estratégias preventivas para } \\
\text { evitar a infecção por COVID-19. }\end{array}$ & $\begin{array}{l}\text { Recomendações baseadas no } \\
\text { Guia Provisório para } \\
\text { Profissionais de Saúde, } \\
\text { elaborado pelo CDC. }\end{array}$ \\
\hline
\end{tabular}

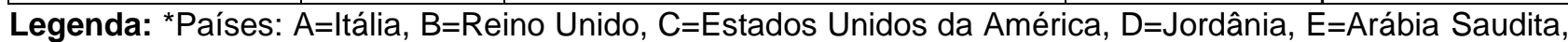
Reino Unido, Brasil, Estados Unidos da América, F=Brasil, G=Arábia Saudita, H=Canadá, I=China, J=Canadá e Brasil. Fonte: Santos KF e Barbosa M, 2020. 


\section{DISCUSSÃO}

Os profissionais de odontologia tiveram que tratar somente urgências e emergências odontológicas, como forma de prevenção e proteção, devido à preocupação com a contaminação da COVID-19 que se espalha pelas dispersões dos aerossóis. Neste contexto, a ausência de informações adequadas sobre a forma de contaminação, limpeza de superfícies com produtos específicos, em ambiente odontológico, distanciamento social e biossegurança rigorosa não eram tão claras, e medidas foram tomadas, a fim de se evitar a disseminação da pandemia (SANTOS KF e BARBOSA M, 2020).

Cada país adotou formas diferentes, no atendimento odontológico, mesmo em fases de pico da Infecção humana ampla. Houve variação nas escolhas de medidas de triagem e nas medidas que poderiam aumentar a infecção cruzada nos consultórios e contribuir para conter a rapidez de propagação do vírus, como em Cingapura. Já o Reino Unido, optou por selecionar os casos, por telefone e atender em centros especializados apenas tratamentos básicos (MALLINENI SK, et al., 2020).

A partir de 20 de março de 2020, no Brasil, a Agência Nacional de Vigilância Sanitária (ANVISA) recomendou que somente, os atendimentos odontológicos de urgência e emergência poderiam ser realizados. Quanto aos profissionais da odontologia, da rede pública, de atendimento do SUS (Serviço Nacional de Saúde) foram alocados para ajudar outros profissionais de saúde, na via rápida da COVID-19.

As prestações de serviços, via telessaúde, foram implementadas e regulamentadas, nos diferentes países, a exemplo dos Estados Unidos (EUA) e do Brasil para reduzir a transmissão da doença, facilitar o atendimento e verificar diferentes sintomatologias (MALLINENI SK, et al., 2020).

Em um estudo, na cidade de Wuhan, China, com 99 pacientes mostrou sintomas como febre, tosse, falta de ar, dor muscular, confusão mental, dor de cabeça, dor de garganta, rinorréia, dor no peito, diarreia, náuseas, vômitos e predominância de homens mais velhos com condições de agravos da síndrome respiratória aguda grave (KHURSHID Z, et al., 2020).

A anosmia e a ageusia são a ausência de olfato total ou parcial causada por lesões do nervo olfativo ou obstrução das fossas nasais e a ausência de paladar, respectivamente, que podem ser identificadas em muitos pacientes acometidos pela COVID-19 e passaram a ser reconhecidas pelo CDC, como sintomas principais e podem ajudar os dentistas a identificar a doença mais facilmente (CDC, 2020; LECHIEN JR, et al., 2020).

Na saliva humana há a possibilidade de investigar a presença de COVID-19, SARS-COV, MERS, ZIKV e outros vírus. Os testes rápidos possuem tecnologia econômica, fácil, não invasiva e precisa de poucos equipamentos para se realizar a testagem. Disponibilizar em locais de fácil acesso a população, como balcões de aeroporto, no check-in, áreas de imigração, hospitais e clínicas voltadas a este tipo de atendimento devem minimizar a transmissão nosocomial e ajudar também trabalhadores da saúde (KHURSHID Z, et al., 2020).

Para Khurshid Z, et al. (2020), os centros de pesquisa, agências de saúde, prestadores de serviços e dentistas devem explorar a oportunidade de diagnóstico do teste precoce salivar, desenvolver ensaios moleculares automatizados para facilitar o atendimento, ajudar na investigação de casos de COVID-19, além de facilitar a quarentena e minimizar danos financeiros aos profissionais.

Reflexões e ações, baseadas em conhecimento das histórias das epidemias são necessárias para garantir a melhor maneira de atuação, diante dos desafios da pandemia da COVID-19 (MARTELLI-JÚNIOR H, et al., 2020). A articulação entre os profissionais de saúde bucal e a equipe de Atenção Primária à Saúde, no processo de triagem, colabora para a organização do serviço de atenção à saúde e diminui exposição de pessoas com usuários sintomáticos da COVID-19 (BRASIL, 2020).

A necessidade de atendimento urgente organizado e realizado em diversos países da pandemia com equipamentos de proteção individual adequados continua sendo prioridade pelos profissionais da odontologia que também, podem contribuir para os cuidados médicos, mesmo que os serviços clínicos prestados, a população, não sejam de forma direta, pois os dentistas sentem um dever moral de reduzir os cuidados de rotina por medo de espalhar a COVID-19 entre seus pacientes e se encontram preocupados com as consequências financeiras (COULTHARD P, 2020). 
No estudo de Wu M e Chang YC (2020), na cidade de Taiwan, foram tomadas abordagens como verificar temperatura acima de $37,5^{\circ} \mathrm{C}$, seguida de questionário com características que continham histórico de viagens, ocupação, histórico de contatos, histórico de clusters e se apresentaram sintomas da infecção, com a finalidade de rastrear pacientes potenciais portadores da COVID-19. Como a propagação do vírus e a transmissão da doença se dá por meio de aerossóis, contato com fluidos humanos e superfícies contaminadas, os profissionais de odontologia e pacientes acabam tendo um risco aumentado, levando a tratamentos emergenciais e consultas online para maior benefício de todos. (WU M e CHANG YC, 2020).

Para as Nações Unidas durante a pandemia do COVID-19, os dentistas devem aceitar no consultório particular apenas urgências não deferíveis, como um abscesso ou pulpite irreversível. Problemas ortodônticos, como problemas gerais de odontologia, representam urgências, não emergenciais verdadeiras; portanto, uma vídeo-chamada (teleodontologia) ou mensagens com fotos pode ser uma opção para avaliar o caso e decidir o melhor momento de tratamento (CASAMASSIMO PS, et al., 2020).

$\mathrm{Na}$ área de Odontopediatria, as urgências devem ser bem orientadas, pois as crianças infectadas podem transmitir a doença aos profissionais da saúde e uma lista preventiva no atendimento às crianças deve ser seguida, além de conter informações com medidas administrativas, educação e treinamento em prevenção de infecções, segurança do pessoal auxiliar da clínica, avaliação de programas educativos na odontopediatria, higiene das mãos, equipamento de proteção individual (EPI), etiqueta de higiene respiratória/tosse, segurança de perfurocortantes, práticas de injeção segura para evitar acidentes, esterilização e desinfecção de itens de forma segura, dispositivos de assistência ao paciente, prevenção e controle ambiental de infecções e qualidade da água da unidade odontológica (MALLINENI SK, et al., 2020).

Em atendimentos odontológicos emergenciais que não caibam o uso de isolamento absoluto, feito com diques de borracha e colocados em dentes individualizados, está indicado o uso de enxaguatório bucal como um pré-procedimento e deve ser feito com agentes oxidantes como peróxido de hidrogênio $\left(\mathrm{H}_{2} \mathrm{O}_{2}\right)$ ou povidine, pois o COVID-19 é um vírus vulnerável à oxidação e este procedimento diminui seu potencial carreador (PENG X, 2020).

Caruso AA, et al. (2020) difundem que o peróxido de hidrogênio $\left(\mathrm{H}_{2} \mathrm{O}_{2}\right)$ é amplamente utilizado como desinfetante cirúrgico ambiental e como desinfetante oral, em odontologia, no tratamento da gengivite e a diferença está na concentração entre eles. Para os métodos de higienização do ambiente, com inativação do coronavírus em superfícies inanimadas é indicado, o uso de $0,5 \%$ de peróxido de hidrogênio $\left(\mathrm{H}_{2} \mathrm{O}_{2}\right)$ em 1 minuto.

O uso de peróxido de hidrogênio a 3\% (10 volumes) não somente pelas propriedades oxidativas e sua remoção mecânica, mas, principalmente, pela indução da resposta inflamatória antiviral natural. Ajudando a impedir a progressão geral da doença das vias aéreas superiores para o trato respiratório inferior. Lavagens nasais e orais, em forma de gargarejo, com $\mathrm{H}_{2} \mathrm{O}_{2}$ a $3 \%$ (10 volumes) devem ser realizadas imediatamente, desde o início dos primeiros sintomas, com a confirmação do diagnóstico, durante o período da doença, em quarentena domiciliar ou em pacientes hospitalizados que não necessitem de tratamento intensivo (CARUSO AA, et al., 2020). As escolas de Odontologia precisam rever seus papéis de atuação, na intenção de expandir a aprendizagem, incluindo funções aos profissionais, que aumentem sua capacitação em momentos de desastres naturais e pandemias.

É importante que as associações de classe da odontologia forneçam educação continuada, para os dentistas e a equipe de saúde bucal e ter como base os princípios de assistência médica aguda, suporte de vida, flebotomia e orientações na prescrição medicamentosa, para minimizar os efeitos da Pandemia (ODEH ND, et al., 2020). Para Peng X, et al. (2020) a transmissão da doença COVID-19 se dá por partículas aerossolizadas, no ar e é tida como a principal via de disseminação, particularmente em clínicas e hospitais dentários.

Recomenda-se para todos os prestadores de serviços de saúde, o uso de EPI como barreira protetiva, incluindo óculos de proteção, máscaras, luvas, bonés, protetores faciais além, de roupas adequadas de proteção que são sempre indicadas em ambientes clínicos/hospitalares, durante o período da Pandemia e, também, verificar os diferentes níveis de biossegurança. 
De acordo, com o Occupational Safety and Health Administration (OSHA)(2020) e Peng X, et al. (2020), há três níveis de recomendações de proteção envolvendo o uso de EPI para profissionais da odontologia, durante o enfrentamento da COVID-19. No nível primário, em que haja procedimentos, sem gerações de aerossol, e em pacientes sem suspeita da doença, cabem o uso de avental comum, como roupas de trabalho, luvas descartáveis de látex ou nitrilo, óculos de proteção ou proteção facial descartável (viseira) e uso de máscara cirúrgica.

Nível secundário, em que haja procedimentos envolvendo geração de aerossóis e sem suspeita da doença cabem as paramentações anteriores, seguidas de avental descartável por cima do avental comum, touca médica descartável e uso de máscara facial N95 ou outras de característica melhor. A N95 é uma máscara que remove $95 \%$ de todas as partículas que tenham menos que 0,3 mícron de diâmetro.

Em casos, de suspeita de paciente portador da COVID-19 e em tratamentos que não envolvam a geração de aerossol cabem, também as recomendações de nível secundário. Recomendações de nível terciário estão voltadas à pacientes com suspeita ou confirmação da doença, embora um paciente portador da doença, não necessariamente, procure tratamento odontológico, mas as recomendações devem ser seguidas de acordo com o protocolo determinado pela OSHA e seguem associadas às recomendações de nível secundário, porém com roupas de proteção extra, descartáveis e capas impermeáveis para os sapatos (OSHA, 2020; PENG X, et al., 2020).

As máscaras de proteção ou respiradores de partículas obedecem às normas americanas e possuem modelos ( $N, R$ e $P$ ), onde $N$ indica que a máscara não é resistente a óleo, $R$ é resistente a óleo e $P$ é a prova de óleo filtrando, pelo menos, $95 \%$ das partículas transportadas pelo ar quando utilizadas de forma adequada (ANVISA, 2009). A R95 é uma peça facial de filtragem que funciona como um respirador para partículas, possui uma válvula com camada de carvão ativado e está indicada como proteção de nível terciário.

Indica-se também, em casos de procedimentos geradores de aerossol, o uso da P95 que é um respirador facial, com ajuste seguro para o profissional, filtra também, pelo menos, 95\% das partículas transportadas pelo ar e é à prova de óleo. Ao se remover o EPI contaminado é recomendado usar uma máscara N95, e não tocar na parte externa da máscara sem o uso de luvas (PENG X, et al., 2020).

\section{CONSIDERAÇÕES FINAIS}

Este artigo evidencia medidas preventivas recomendadas para diminuir o risco de infecção nos profissionais atuantes, no ambiente odontológico e nas urgências e emergências, para controlar as vias de transmissão da COVID-19 e evitar contaminações cruzadas. Elucida que os cirurgiões-dentistas podem contribuir com suas expertises, neste momento crítico de pandemia, pois trabalham clinicamente em ambulatórios públicos ou privados e devem usar paramentação biossegura, fazer uso de termômetros digitais para aferição de temperatura maior que $37,5^{\circ} \mathrm{C}$. A indicação e treino aos testes orais salivares e testes rápidos sorológicos, realizados pelos dentistas, podem ser uma prática inserida como forma preventiva e ajudar outros profissionais da saúde, no combate à pandemia.

\section{REFERÊNCIAS}

1. AGÊNCIA NACIONAL DE VIGILÂNCIA SANITÁRIA (ANVISA). Cartilha de proteção respiratória contra agentes biológicos para trabalhadores de saúde. Brasília: Anvisa, 2009.

2. BERLIN-BRONER Y, LEVIN L. Dental hierarchy of needs' in the COVID-19 era - or why treat when it doesn't hurt? Oral Health Prev Dent, 2020; 18(2): 95.

3. BRASIL. Ministério da Saúde, ORGANIZAÇÃO PAN-AMERICANA DA SAÚDE. Biossegurança em saúde: prioridades e estratégias de ação. Brasília: Ministério da Saúde, 2010.

4. BRASIL. Ministério da Saúde. Secretaria de Atenção Primária à Saúde. Atendimento odontológico no SUS: nota técnica no 9/2020-CGSB/DESF/SAPS/MS. Brasília: MS, 2020. (Coronavírus, COVID-19).

5. CAPRIOGLIO A, et al. Management of orthodontic emergencies during 2019-NCOV. Prog Orthod, 2020 Apr 7; 21(1): 10.

6. CARUSO AA, et al. May hydrogen peroxide reduce the hospitalization rate and complications of SARS-CoV-2 infection? Infect Control Hosp Epidemiol, 2020 Apr 22: 1-2. doi: 10.1017/ice.2020.170. [Epub ahead of print]. 
7. CASAMASSIMO PS, et al. Pediatric dentistry during and after COVID-19. Pediatr Dent, 2020 Mar 15; 42(2): 87-90.

8. CENTERS FOR DISEASE CONTROL AND PREVENTION (CDC). Symptoms of coronavirus [Internet]. Page last reviewed: March 20, 2020 [cited 2020 Apr 30]. Available from: https://www.cdc.gov/coronavirus/2019-ncov/symptomstesting/symptoms.html.

9. COULTHARD P. Dentistry and coronavirus (COVID-19): moral decision-making. Br Dent J, 2020 Apr; $228(7)$ : 503-505.

10. FAROOQ I, ALI S. COVID-19 outbreak and its monetary implications for dental practices, hospitals and healthcare workers. Postgrad Med J, 2020 Apr 3 [cited 2020 Apr 16]. pii: postgradmedj-2020-137781. Available from: doi: 10.1136/postgradmedj-2020-137781. [Epub ahead of print].

11. JOHNS HOPKINS UNIVERSITY. COVID-19 Dashboard by the Center for Systems Science and Engineering (CSSE) at Johns Hopkins University (JHU) [Internet]. 2020 [cited 2020 May 12]. Available from: https://gisanddata.maps.arcgis.com/apps/opsdashboard/index.html\#/bda7594740fd40299423467b48e9ecf6.

12. KAMPF G, et al. Persistence of coronaviruses on inanimate surfaces and their inactivation with biocidal agents. J Hosp Infect, 2020 Mar; 104(3): 246-251.

13. KHADER Y, et al. Dentists' awareness, perception, and attitude regarding covid-19 and infection control: crosssectional study among jordanian dentists. JMIR Public Health Surveill, 2020 Apr 9; 6(2): e18798.

14. KHURSHID Z, et al. Human saliva: non-invasive fluid for detecting novel coronavirus (2019-nCoV). Int J Environ Res Public Health, 2020 Mar 26 [cited 2020 Apr 16]; 17(7). pii: E2225. Available from: doi: 10.3390/ijerph17072225.

15. LECHIEN JR, et al. Olfactory and gustatory dysfunctions as a clinical presentation of mild-to-moderate forms of the coronavirus disease (COVID-19): a multicenter European study. Eur Arch Otorhinolaryngol [ínternet], 2020 Aug [cited 2020 Aug 30]; 277(8): 2251-2261. Available from: https://link.springer.com/article/10.1007/s00405-020-05965-1.

16. MALLINENI SK, et al. Coronavirus disease (COVID-19): characteristics in children and considerations for dentists providing their care. Int J Paediatr Dent, 2020 May; 30(3): 245-250.

17. MARTELLI-JÚNIOR H, et al. Dental journals and coronavirus disease (COVID-19): a current view. Oral Oncol [Internet], 2020 Jul [cited 2020 Jul 30]: 104664. Available from: doi: 10.1016/j.oraloncology.2020.104664.

18. MENG L, et al. Coronavirus disease 2019 (COVID-19): emerging and future challenges for dental and oral medicine. J Dent Res [Internet], 2020 Mar [cited 2020 Apr 16]; 99(5): 481-487. Available from: https://doi.org/10.1177/0022034520914246.

19. OCCUPATIONAL SAFETY AND HEALTH ADMINISTRATION (OSHA). COVID-19: control and prevention: dentistry workers and employers [Internet]. Washington, DC, [2020; cited 2020 May 6]. Available from: https://www.osha.gov/SLTC/covid-19/dentistry.html.

20. ODEH ND, et al. COVID-19: Present and future challenges for dental practice. Int J Environ Res Public Health [Internet], 2020 Apr 30 [cited 2020 May 6]; 17(9): E3151 [10 pages]. Available from: https://www.mdpi.com/1660-4601/17/9/3151.

21. PENG X, et al. Transmission routes of 2019-nCoV and controls in dental practice. Int J Oral Sci, 2020 Mar 3; $12(1)$ : 9.

22. PRATI C, et al. COVID-19: its impact on dental schools in Italy, clinical problems in endodontic therapy and general considerations. Int Endod J 2020 May; 53(5): 723-725.

23. SABINO-SILVA R, et al. Coronavirus COVID-19 impacts to dentistry and potential salivary diagnosis. Clin Oral Investig, 2020 Apr; 24(4): 1619-1621.

24. SANTOS KF, BARBOSA M. COVID-19 e a odontologia na prática atual. Scielo Preprints [Preprint]. 2020 [postado 12 jun 2020; citado 20 ago 2020]: [25 p.]. DOI: https://doi.org/10.1590/SciELOPreprints.746.

25. SPAGNUOLO G, et al. COVID-19 outbreak: an overview on dentistry. Int J Environ Res Public Health, 2020 Mar; 17(6): 2094.

26. TO KKW, et al. Consistent detection of 2019 novel coronavirus in saliva. Clin Infect Dis, 2020 Aug [cited 2020 Aug 16]; 71(15): 841-843. Available from: https://doi.org/10.1093/cid/ciaa149.

27. VAN DOREMALEN N, et al. Aerosol and surface stability of SARS-CoV-2 as compared with SARS-CoV-1. N Engl J Med [serial online], $2020 \mathrm{Apr} 16$ [cited 2020 May 6]; 382(16): 1564-1567. Available from: https://www.nejm.org/doi/pdf/10.1056/NEJMc2004973?articleTools=true.

28. WORLD HEALTH ORGANIZATION (WHO). Annex G: use of disinfectants: alcohol and bleach. In: World Health Organization. Infection prevention and control of epidemic-and pandemic-prone acute respiratory infections in health care: WHO guideline. Geneva: WHO, 2014. p. 65-66.

29. WORLD HEALTH ORGANIZATION (WHO). Infection prevention and control (IPC) for novel coronavirus (COVID-19) [Internet]. 2020 [cited 2020 Apr 7]. Available from: https://openwho.org/courses/COVID-19-IPC-EN.

30. WU M, CHANG YC. COVID-19 and its implications in the management of resource infrastructure. J Dent Sci [Internet], 2020 Jun [cited 2020 Jun 30]; 15(2): 225-226. Available from: https://www.sciencedirect.com/science/article/pii/S1991790220300763?via\%3Dihub.

31. YANG Y, et al. Experience of diagnosing and managing patients in oral maxillofacial surgery during the prevention and control period of the new coronavirus pneumonia. Chin J Dent Res, 2020; 23(1): 57-62.

32. ZHU N, et al. A novel coronavirus from patients with pneumonia in China, 2019. N Engl J Med, 2020 Feb 20; 382(8): 727-733. 